\title{
الفن التفاعلي يصل بالعمارة الاخلية إلى عالم من الواقع الافتراضي المختلط Interactive art takes interior architecture to a world of mixed virtual reality
}

د. أيمن صلاح الاين حسين الرويني مدرس العمارة الداخلية بقسم الديكور ـ كلية الفنون الجميلة جامعة الإسكندية

تمهيد:

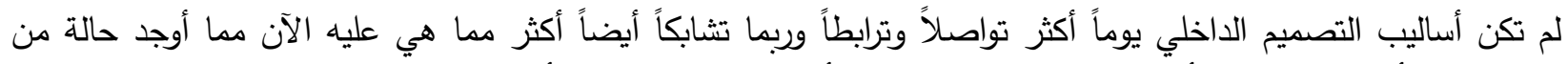

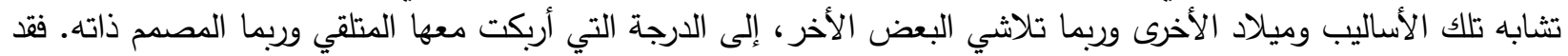

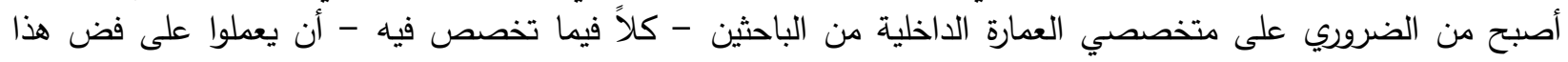

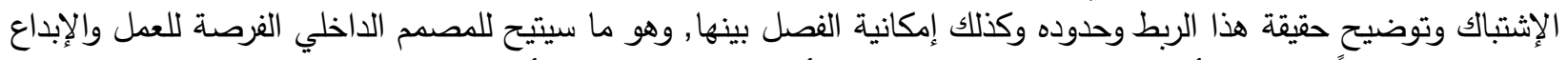

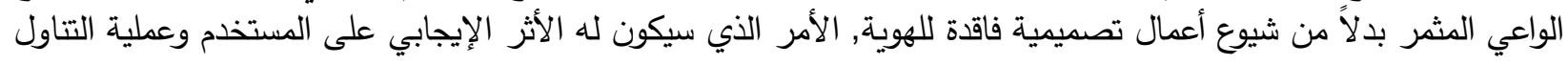
الفراغي برمتها. ويأتي موضوع هذا البحث في إطار هذا المفهوم, إلا أن الباحث يحاول في هذا الصدد التركيز على أثر الفن التفاعلي

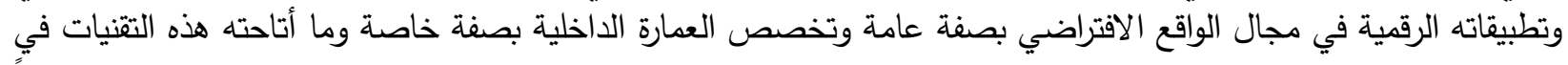

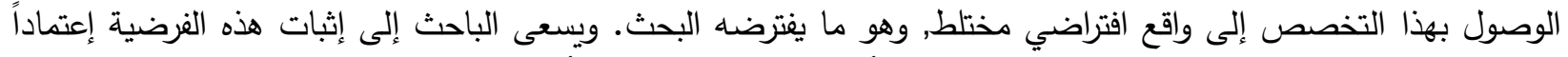

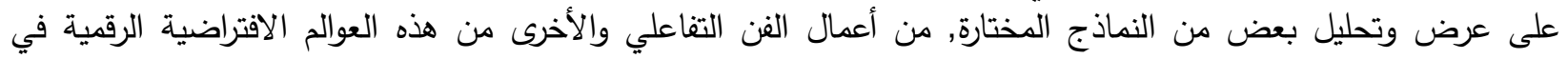

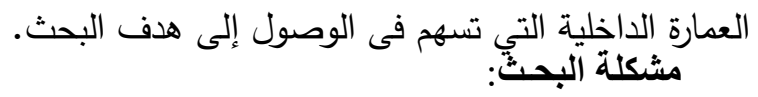

تكمن مشكلة البحث في الإجابة على السؤال التالي:- هل أدى الفن التفاعلي في العمارة الداخلية إلى تحول الشكل الفراغي إلى أن يصبح واقعاً افتراضياً مختلطاً؟ الإباء

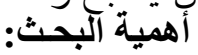

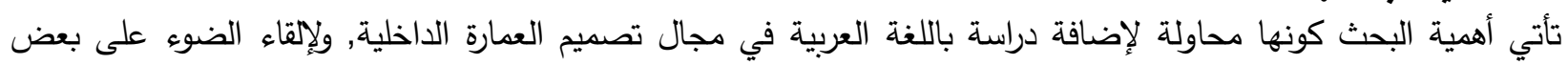

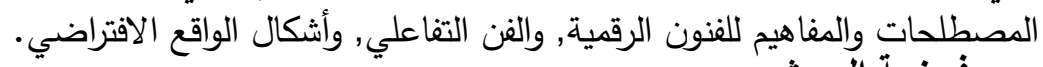
فرضية البحث :

يفترض الباحث أن تطبيق التقنية الرقمية في تصميم وتتفيذ العمارة الداخلية قد أدى إلى إصباغ هذا التخصص بالتفاعلية

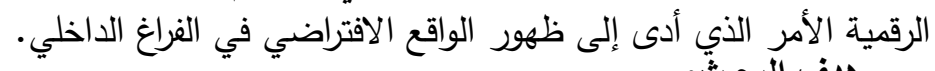

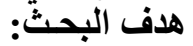
يهدف البحث إلى أثبات فرضية البحث, والإجابة على السؤال الوارد بمشكلة البحث.

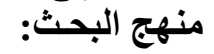
يتخذ الباحث الدنهج الوصفي التحليلي في إجراء هذا البحث.

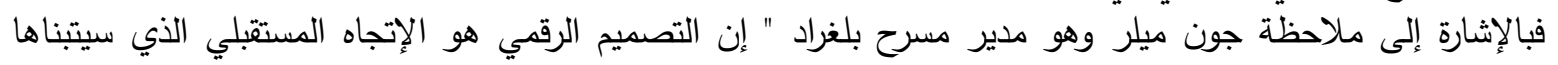

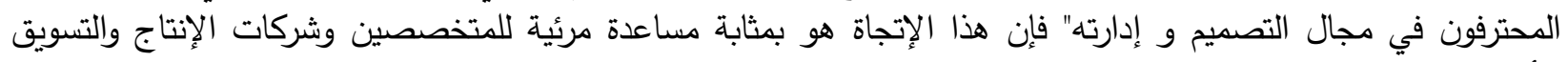
الأمر الذي سيعود بالنفع على الصناعة. فلقد لوحظ أن تطبيق الأساليب التكنولوجية وإبتكار أساليب مستحدثة يتيح لمصمم العمارة الداخلية عمل تصور افتراضي ثلاثي

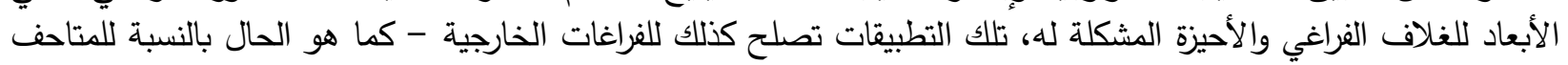

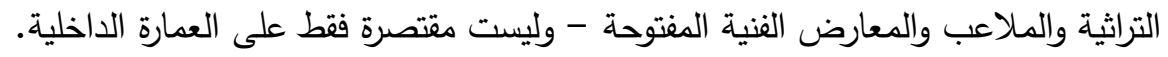

أولاً: الفنـون الرقمية - Digital Arts

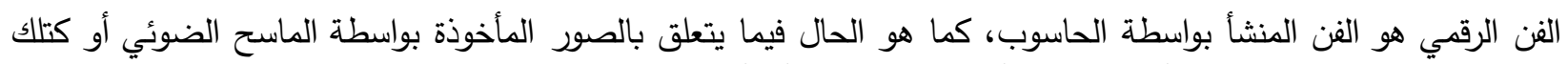

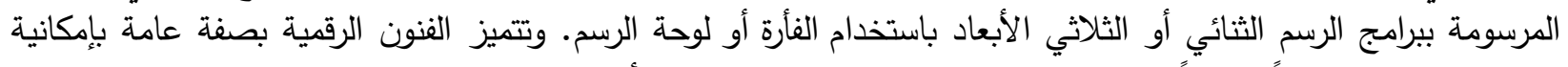

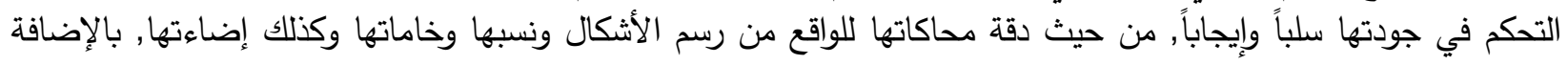

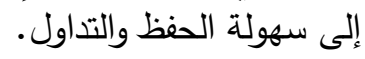


وفي جميع الأحوال فإن تفاوت المنتج النهائى للتصميم الرقمي لا يعتمد فقط على المصمم بل أيضاً وبدرجة أكبر على كفاءة

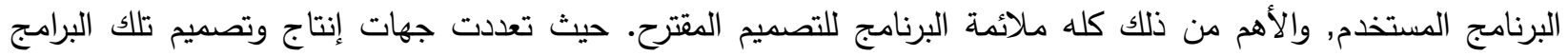

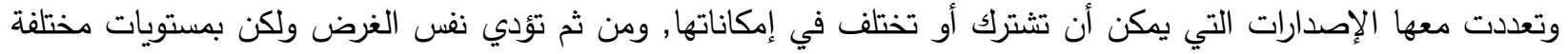

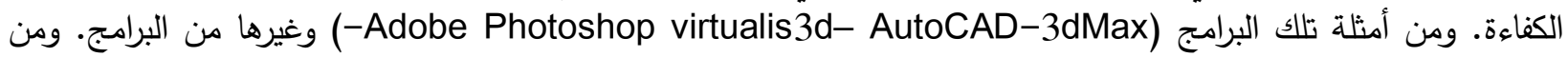

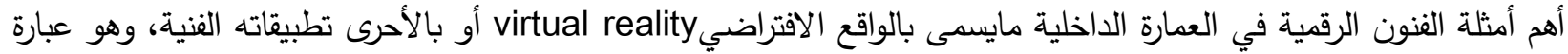

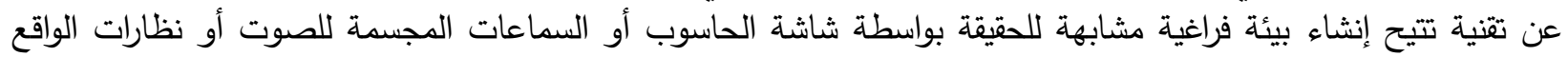

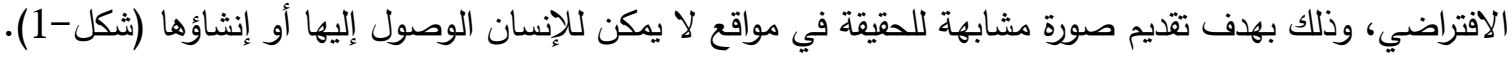
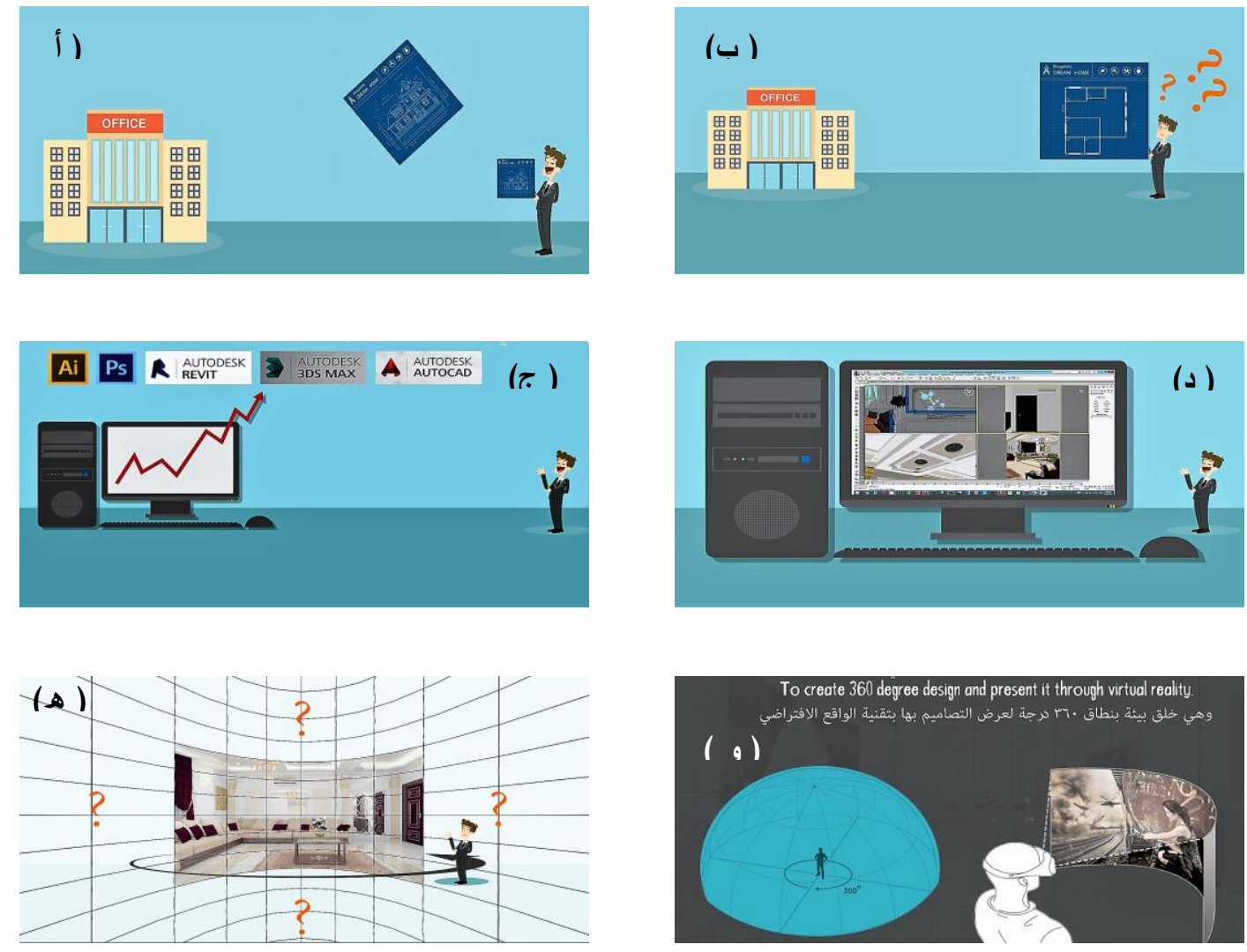

( شكل - 1 ) كان من ثمرة تطور التطبيقات والبرامج الرقمية - من مرحلة التصميم ثنائي الأبعاد إلى الثلاثي

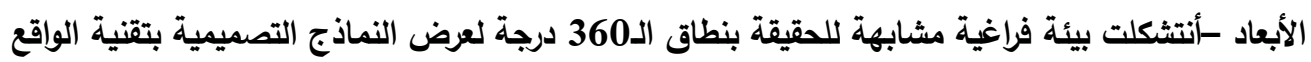

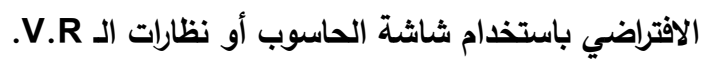

$$
\text { ( أ ) الواقع الافتراضي }
$$

هناك ثلاثة أنواع رئيسية من الواقع الافتراضي يتم الإستعانة بها في أعمال التصميم الداخلي، أو ثلاثة عوالم يخلقها هذا الواقع

Virtual reality completed presence - 1

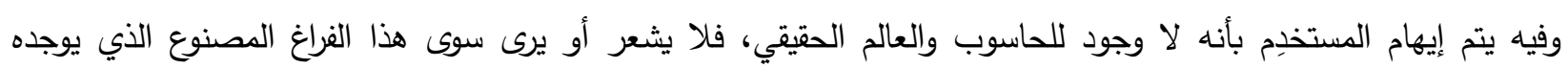

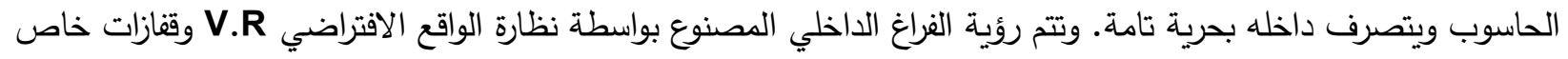




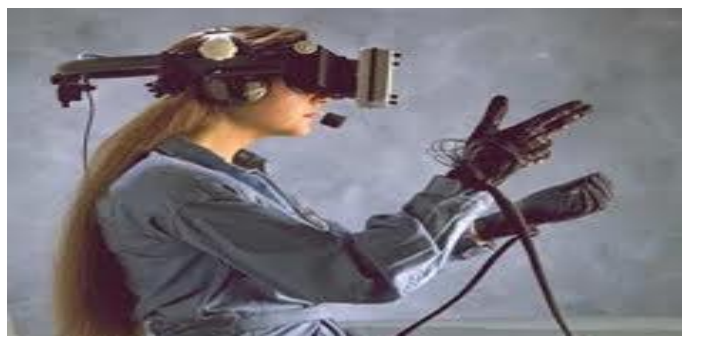
4 ( شكل - 2) كوسيلة إضـافية لتجسيد فراغ (فتراضسي مكتمل التواجد، يتم الإستعانة بنظارة الـ V.Rوالقفازات التى

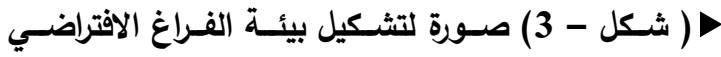
والمكونات الرئيسة له باستخدام برنامج virtualis3d. تستخدم فحى ملامسـة الأسطح وعناصر التأثيث التى يظن

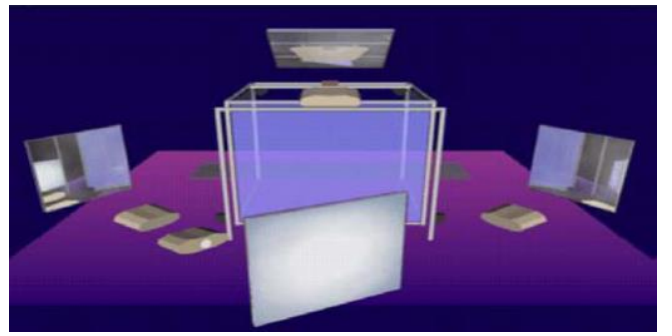

ة تتصل بالحاسوب كوسيلة إضافية لتجسيد الفراغ الافتراضي مما يتيح للمستخدم ملامسة الأسطح وعناصر التأثيث التي يظن

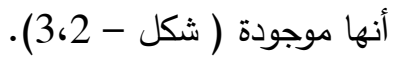

2 - واقع افتراضي محدود الوظيفة والمكان Virtual reality limited function and place

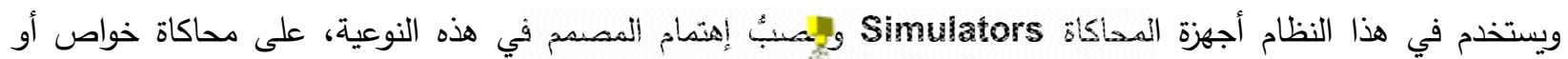

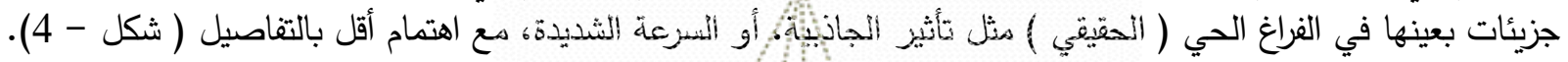
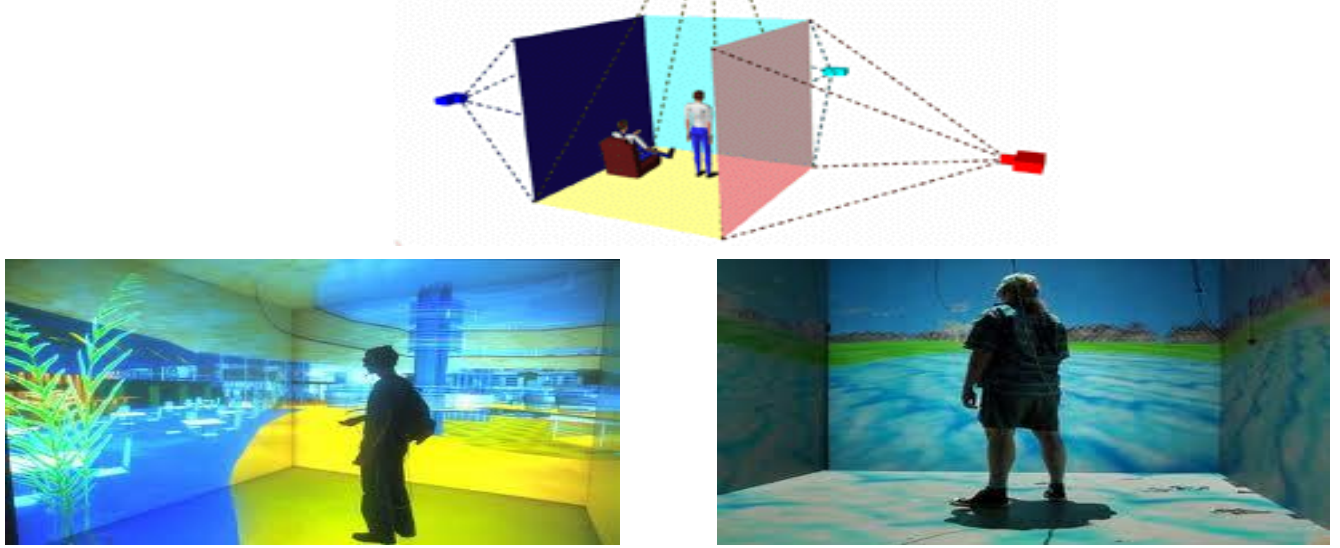

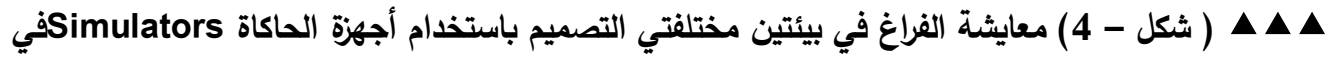

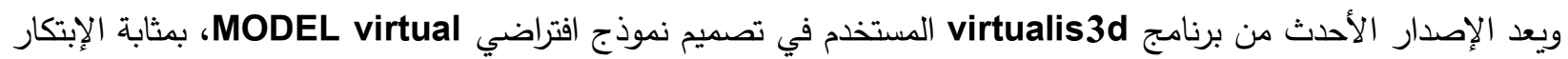

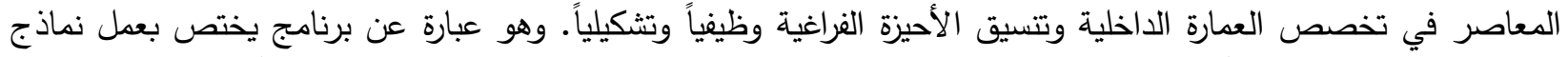

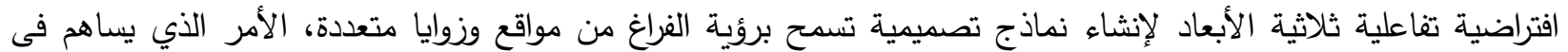

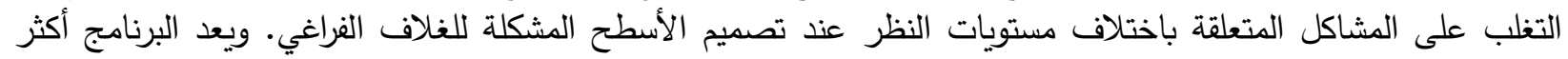

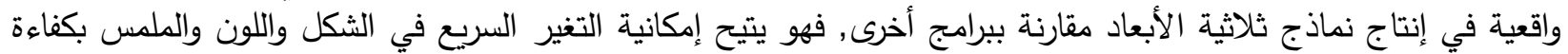

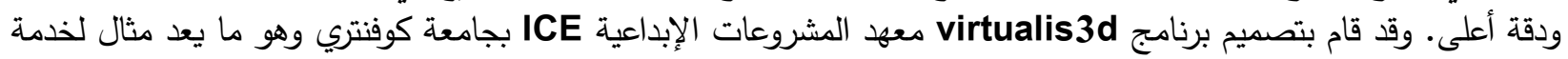

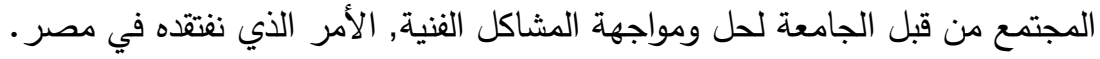

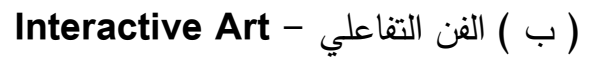

وهو فن يعتمد على التفاعل بين العمل الفني والمتلقي, والفن التفاعلي كإتجاه فكري لا يعد حديثاً فقد ظهر في المسرح الملحمي

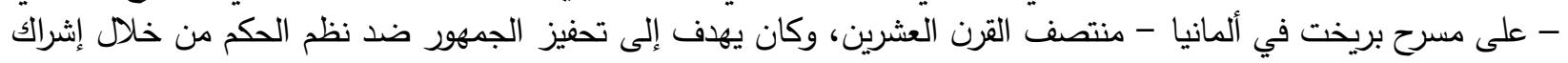




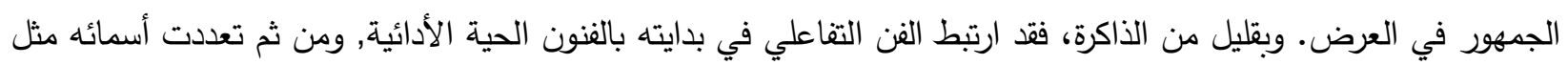

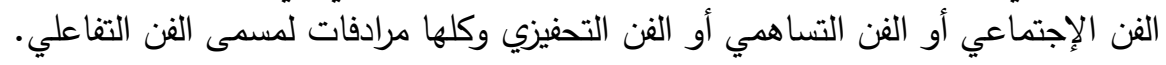

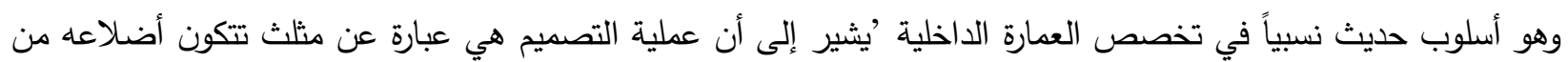

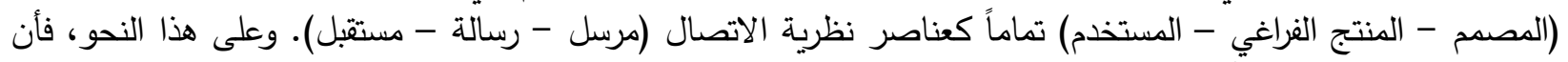

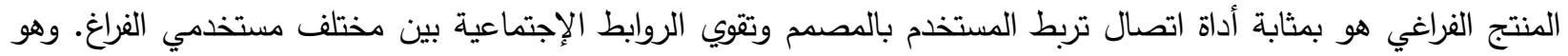

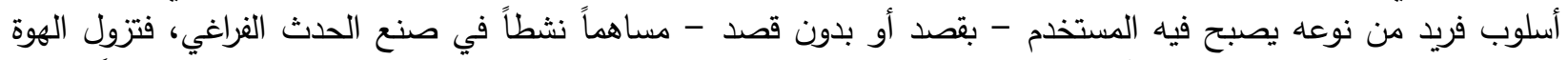

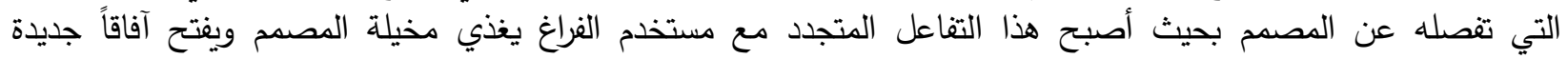

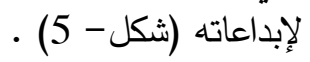

وقد عرف الفن التقاعلي انتشاراً أوسع في العمارة الداخلية المعاصرة، كما هو الحال بالنسبة للفنون الرقمية التي توفر - بفضل

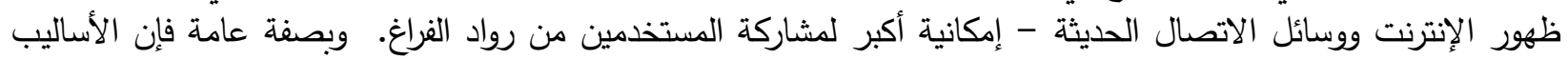

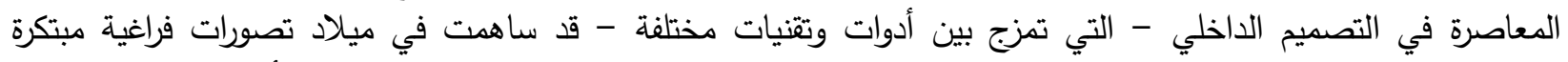

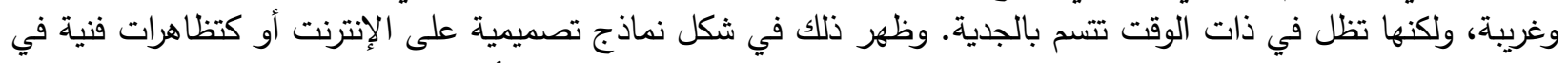

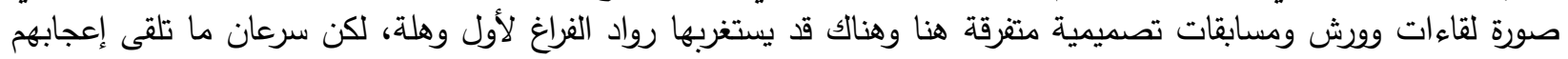

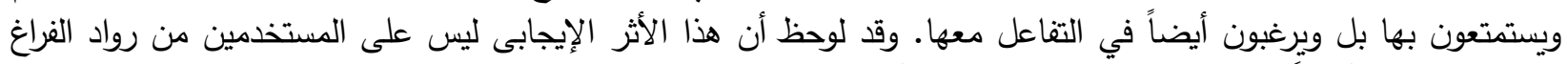

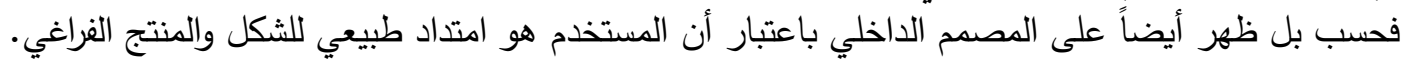
وتوضحح النماج التفاعلية التالية (شكل- 6 ، 7 7) إمكانية توظيف التطبيقات الرقمية الحديثة في إنتاج فراغات داخلية ذات التئية طبيعة تفاعلية لتخلق تلك النماذج علاقة تبادلية بين المصمم والمستخدم، بهدف اكتساب المعرفة في مجال التصميمات الرقمية
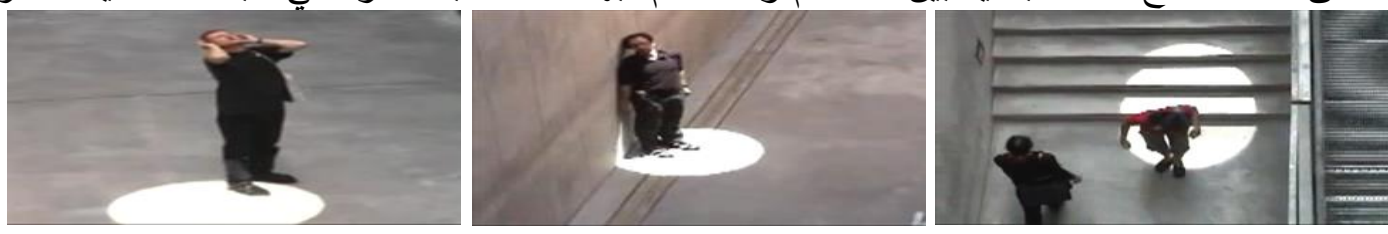

التفاعلية.

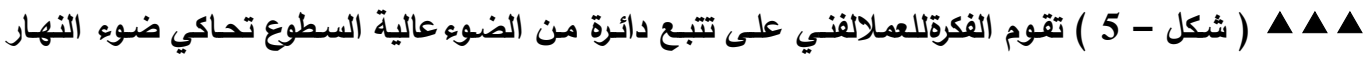

تتفاعل مع الشخصالذييدخل نطاق هذه الدائرة ,حيث يتم تحديده من خلال برنامج على الحاسوب متصل بوحدة
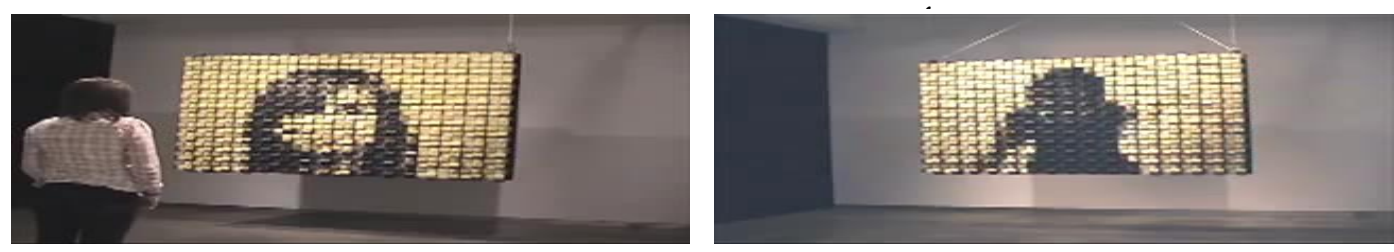

ه $\Delta$ ( شكل - 6 ) صورتان للعمل الفنى التفاعلى Weave Mirror تصميم Daniel Rosin إنتاج 2007، تقوم فكرة العمل على مفاجأة المتلقيلظهور صورة تقربية له على سطح اللوحة - المتموج - الذى يشـاهده, بل
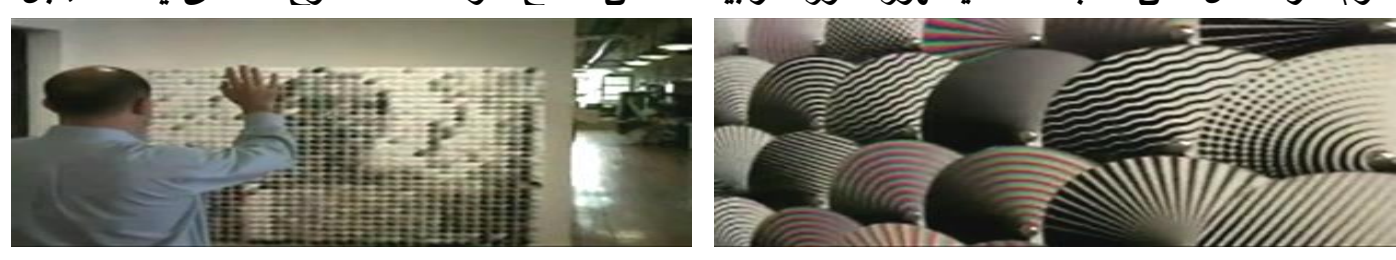

ه 4 ( شكل - 7 ) صورتان للعمل الفنى التفاعلي Daircles Mirror لذات المصمم Daniel Rosin إنتاج 2007، وتقوم فكرة العمل على نفس فكرة العمل السابق من حيث مفاجأة المتلقي حالظهور صورتهانتظاراًتفاعله 
Interactive space - ثانياً: الفراغ التفاعلي تعد الفنون الحية بصفة عامة والعمارة الداخلية بصفة خاصة من الفنون التي يتم تتاولها بالإلتفات والحركة، وذلك بوصفها

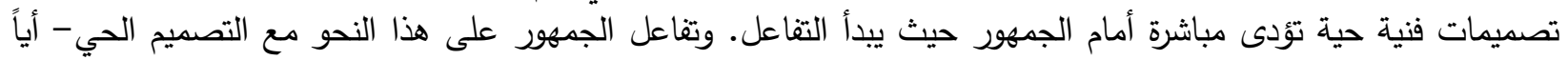

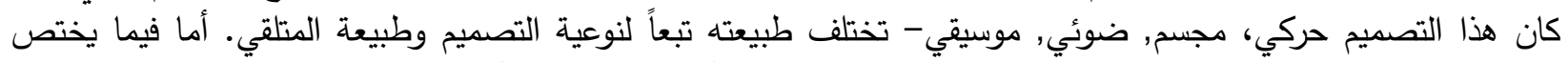

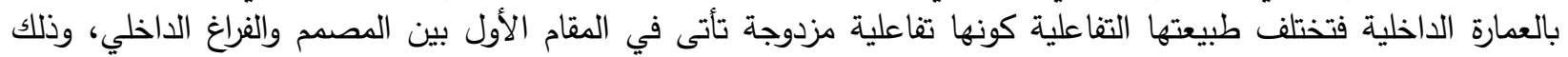

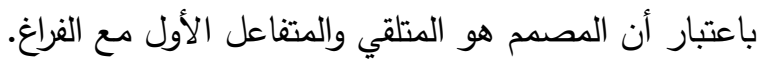
وفي جميع الأحوال يتم الإستعانة بالبرامج ثلاثية الأبعاد لإنتاج التصميمات الحية المشكلة داخل الفراغ التفاعلي، كما هو الحال

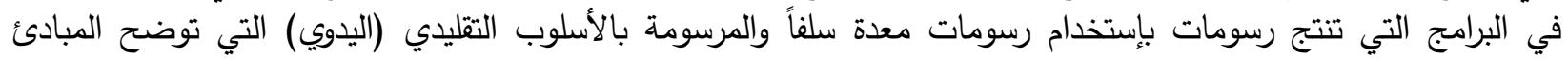

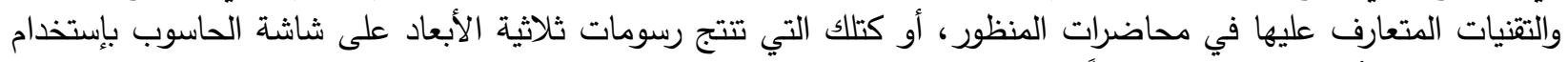

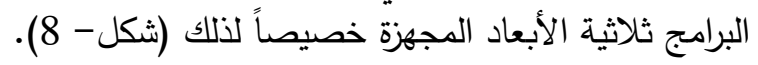
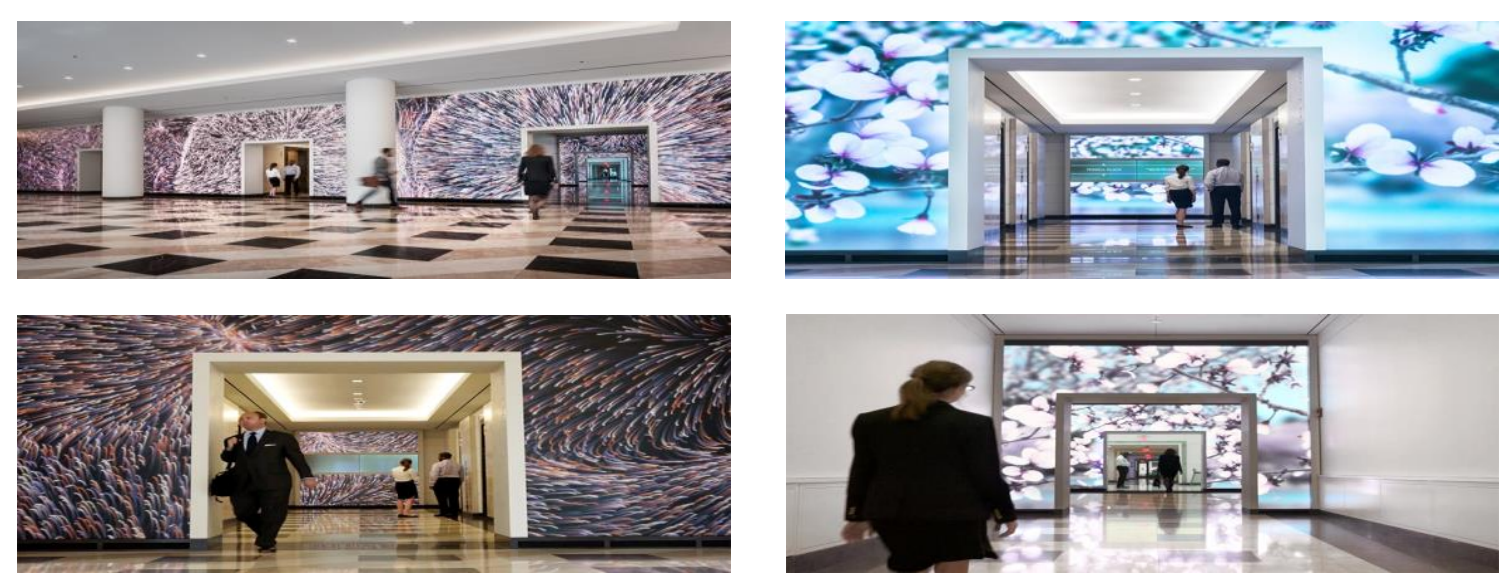

( شكل - 8 ) كوسيلة إضـافية من وسـائل تنشيط الحركة يتم الاستعانة بالبرامج ثلاثية الأبعاد لإنتاج تصميماتتفاعلية

Virtual Space ثالثاً: الفراغ الافتراضي

يهدف الفراغ الافتراضي إلى تقديم نظام مبتكر من عروض الفيديو عالية الجودة, الأمر الذي حقق إمكانية توظيف هذه التقنية

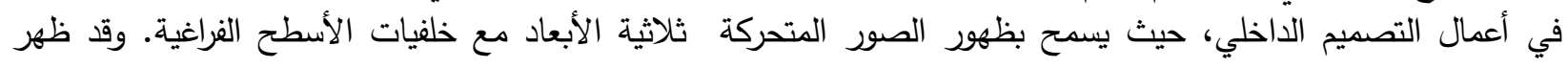

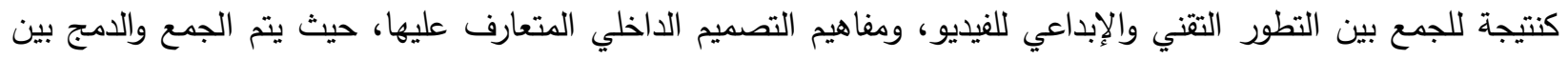
الفيلم والعناصر المادية المكونة للفراغ.

والجزء المادي (الفيزيائي) في الفيلم هو عبارة عن صور متحركة مضيئة تمثل الخلفيات والأسطح الفراغية مع كامل عناصر

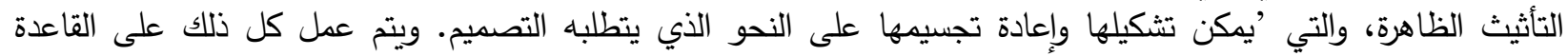

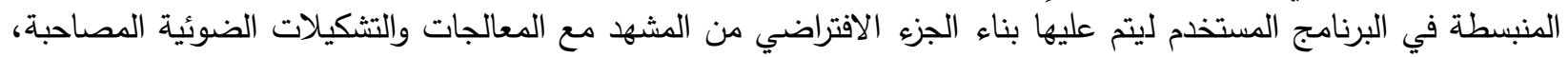

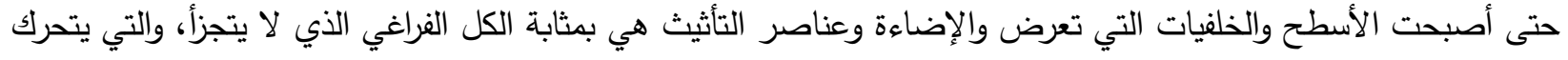
عليها المستخدم كما اعتاد وبشكل طبيعي.

وعلى هذا النحو فإن تجربة الفراغ الحية لم تفقد أيٍ من عناصرها، فقد ساهم استخدام هذه التقنية في أعمال التصميم الداخلي

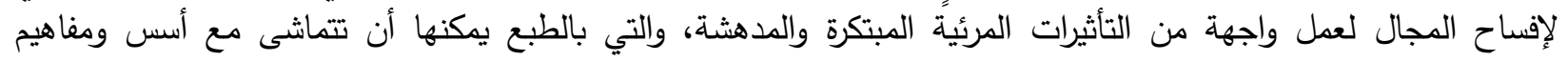

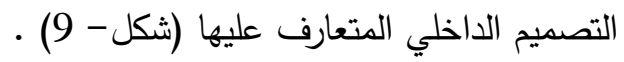

إن تصميم الفراغ الافتراضي يسمح بتقديم أي منهج أو أسلوب فني، مادي كان أو افتراضي, طبيعي أو سيريالي، أو حتى المزج

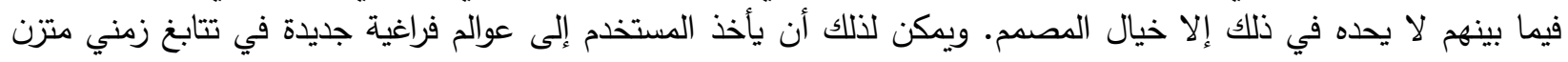




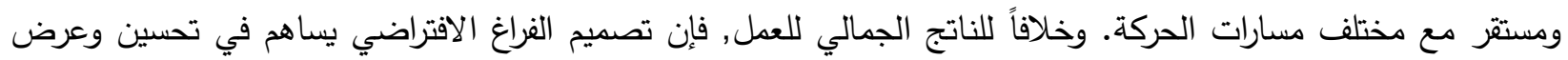
الفكرة بشكل كبير وذلك بإبراز الجوانب التشكيلية والإبداعية مع تحقيق المتطلبات الوظيفية.

( شكل - 9) يكهف الفراغ الافتراضـي إلى تقديم نظام مبتكر من الصور المتحركة ثلاثية الأبعاد للغلافالفراغي، حيثيتم

تثكيل وإعـادة تجسيمكامل عناصر التأثيث الظـاهرة على القاعدة المنبسطة في البرنـامـج المستخدم مـع المعالجـات
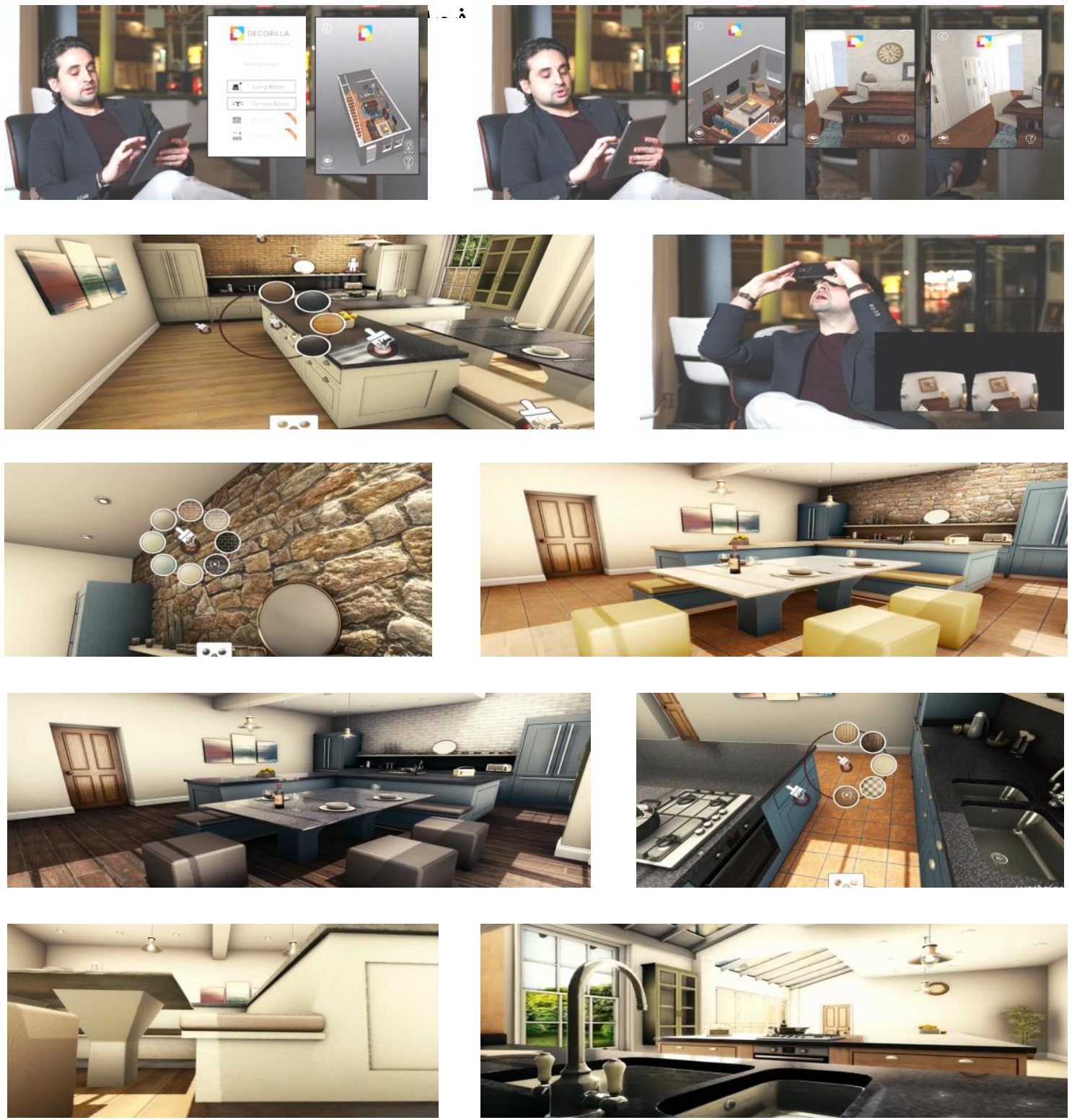

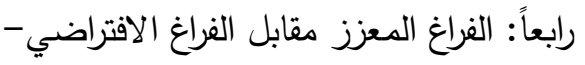

Enhanced space versus interactive space

في الإككان وصف الفراغ المعزز بأنه إحدى التجارب التفاعلية في العمارة الداخلية حيث يتم "تعزيز" العناصر الموجودة في

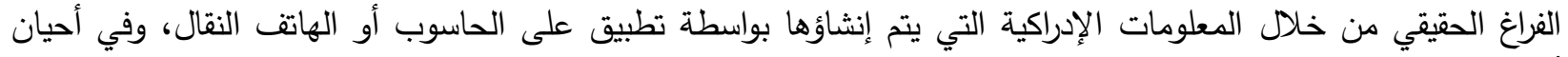

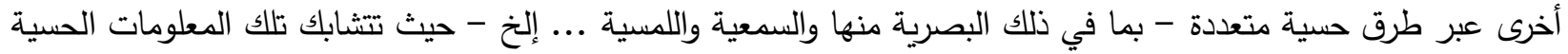


المتراكبة (أي المضافة إلى الفراغ الواقعي) لتغمر وتتساب مع الغلاف الفراغي وعناصر التأثيث المشكلة له. وفي حين يحل

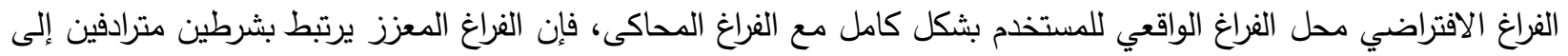

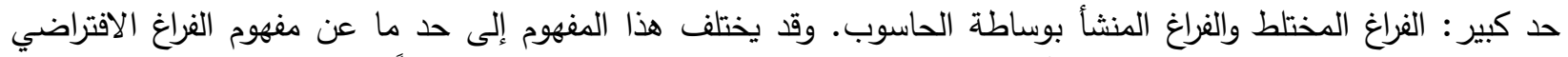
الذي يتم التفاعل معه بواسطة الحاسوب، أما هنا فتضاف الحقيقة المعززة إلى الفراغ المادي بدلاً من استبداله.

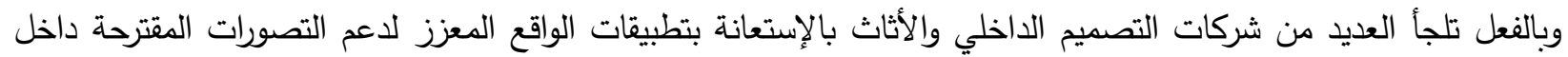

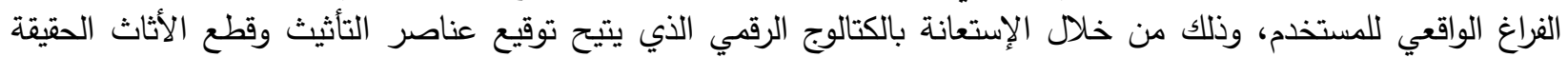

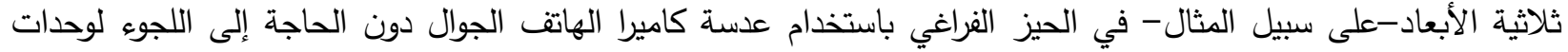

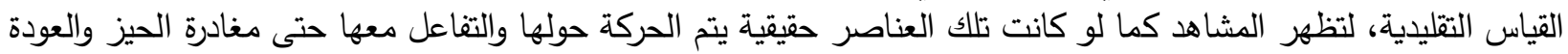

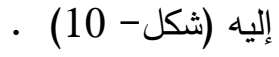
وهذا لا يعني أن الهواتف النقالة أو الأجهزة اللوحية ستكون الوسيلة الوحيدة لإستخدام تقنية الفراغ المعزز ، فهناك العديد من الأجهزة الأخرى القابلة للارتداء والتي أصبح في الإمكان هذه الأيام توظيفها والإستعانة بها.

( شكل - 10 ) من خلال الإستعانة بالكتالوج الرقيسلتقنية الواقع المعزز - يتم توقيع عناصر التأثيثوقطع الأثاث الحقيقةثلاثية|الأبعاد في الحيز الفراغي، باستخدام

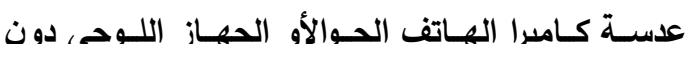
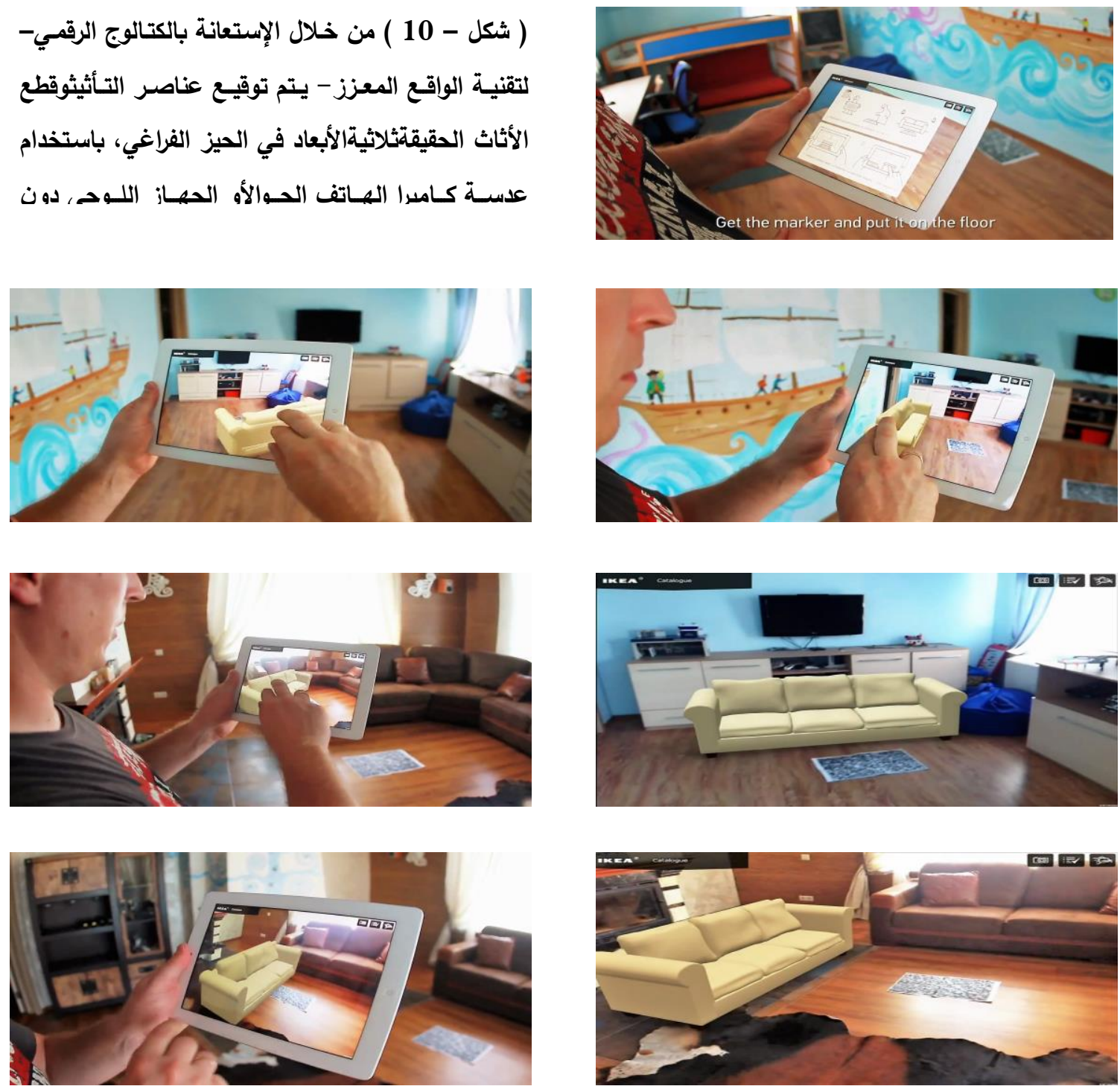
Interactive virtual space - خامساً: الفراغ الافتراضي التفاعلي وهو عبارة عن تقنية تمكن مصمح العمارة الداخلية من إجراء حوار مع أجزاء في العقل الفردي- من خلال الحركة متمثلة في الإلتفات والمشي- وأيضاً كوسيلة للتفاعل مع عقول الآخرين وهي تقنية مستحدثة وهامة لأعمال التصميم الداخلي أتاحها

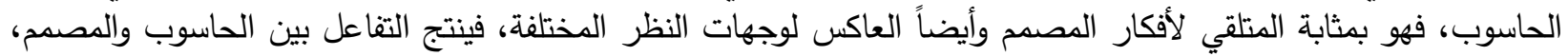

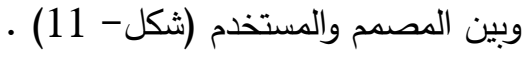

( شكل - 11 ) الواقع الافتراضي، هو عبارة عن تقنية تمكن مصمم العمارة الاخلية من إجراء حوار مع أجزاء في العقل الفردي من خلال الحركة متمثلةفي الإلتفات والمشيمما ينتجنهالتفاعل بين المصمم والحاسوب،فهو بمثابة المتلقي لأفكار المصمم وأيضاً العاكس لوجهات النظر المختلفة. تصميم لنموذجافتراضي تفاعليلإحدى المجمعات السكنية المتكاملة، حيث
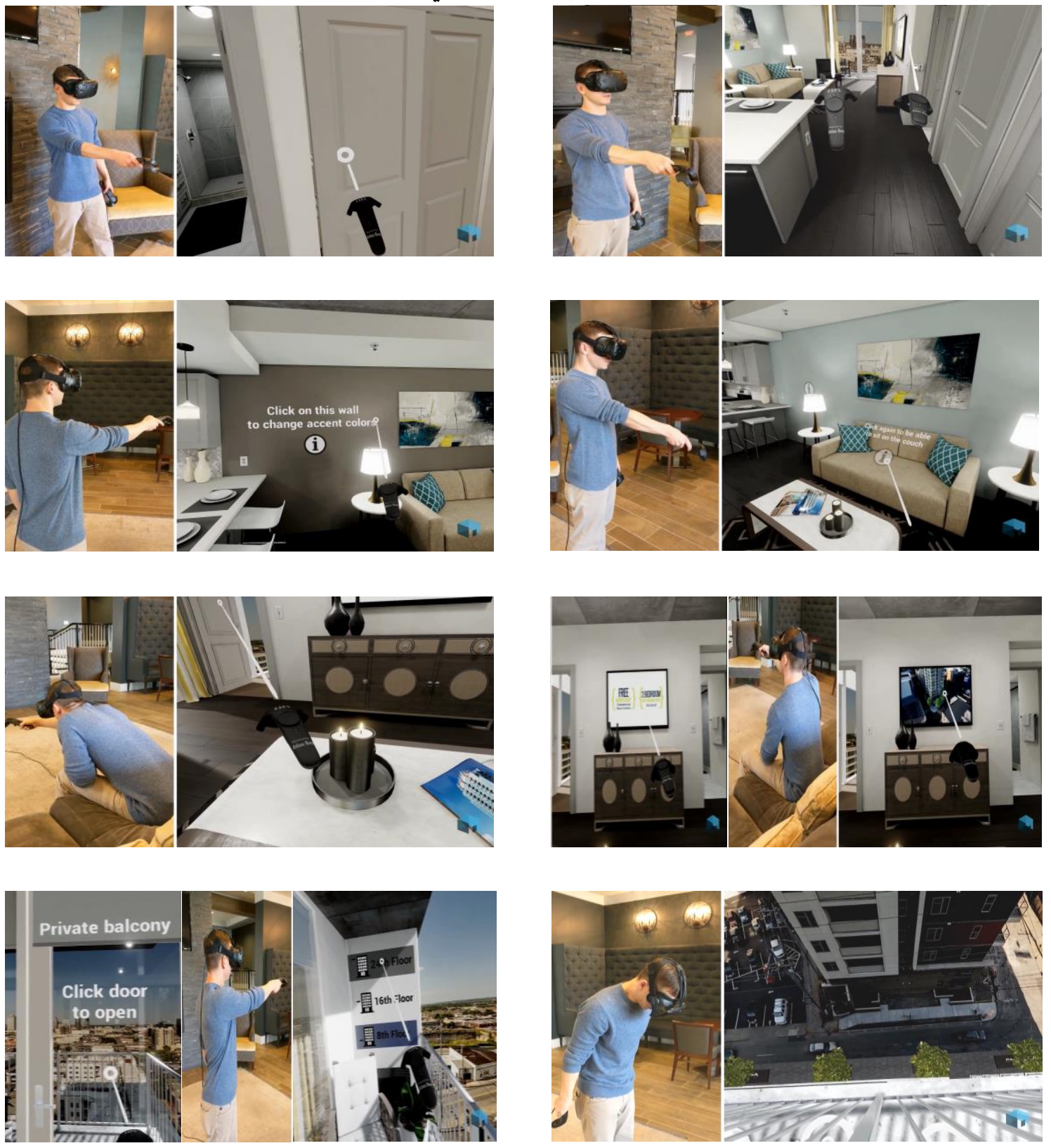
ففي معظم مشاريع التصميم الداخلي يقوم المصمم بجمع المعلومات قبل البدء في عمل النموذج، وعادة ما يتم وضع الأفكار

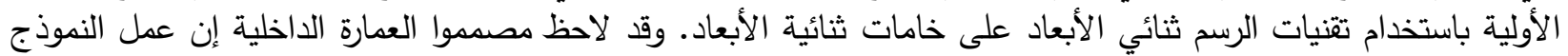

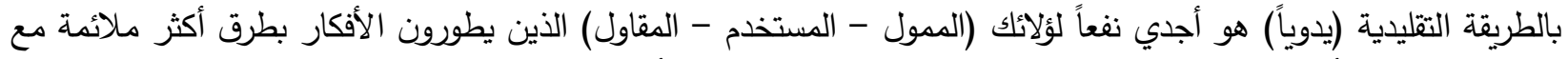

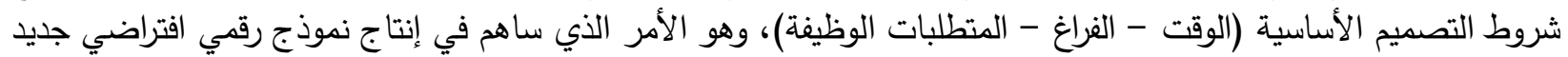

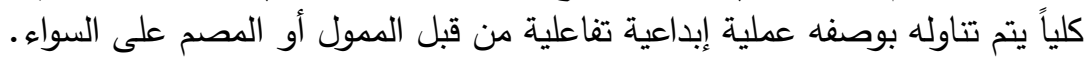

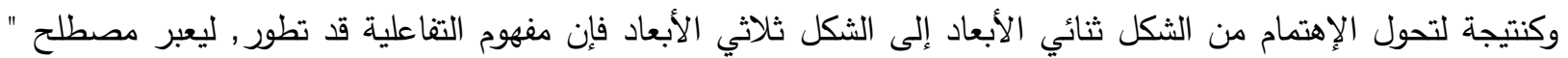
الفراغ الافتراضي التفاعلي " عن الإتجاه المستقبلي في ممارسة العمارة الداخلية (شكل الإن- 12).

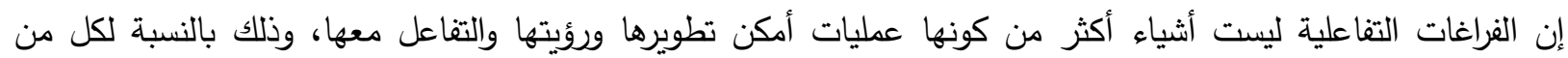

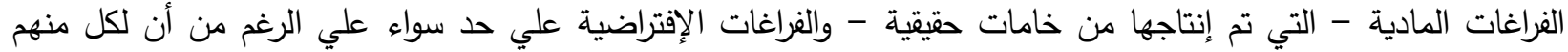

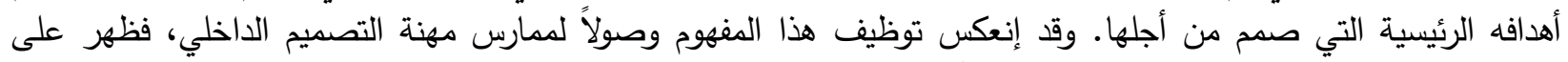

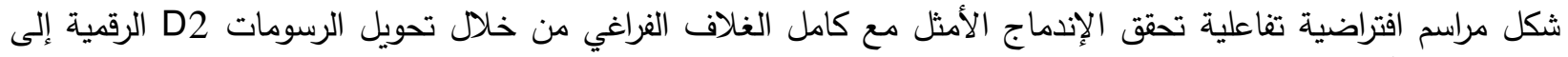
عالم ثلاثي الأبعاد.

( شكل - 12 ) كان من ثمرةتطويرالتقنيات الرقمية في التصميم الداخلي أن ظهر بما يعرف بالمرسم الافتراضي، وهو عبارة
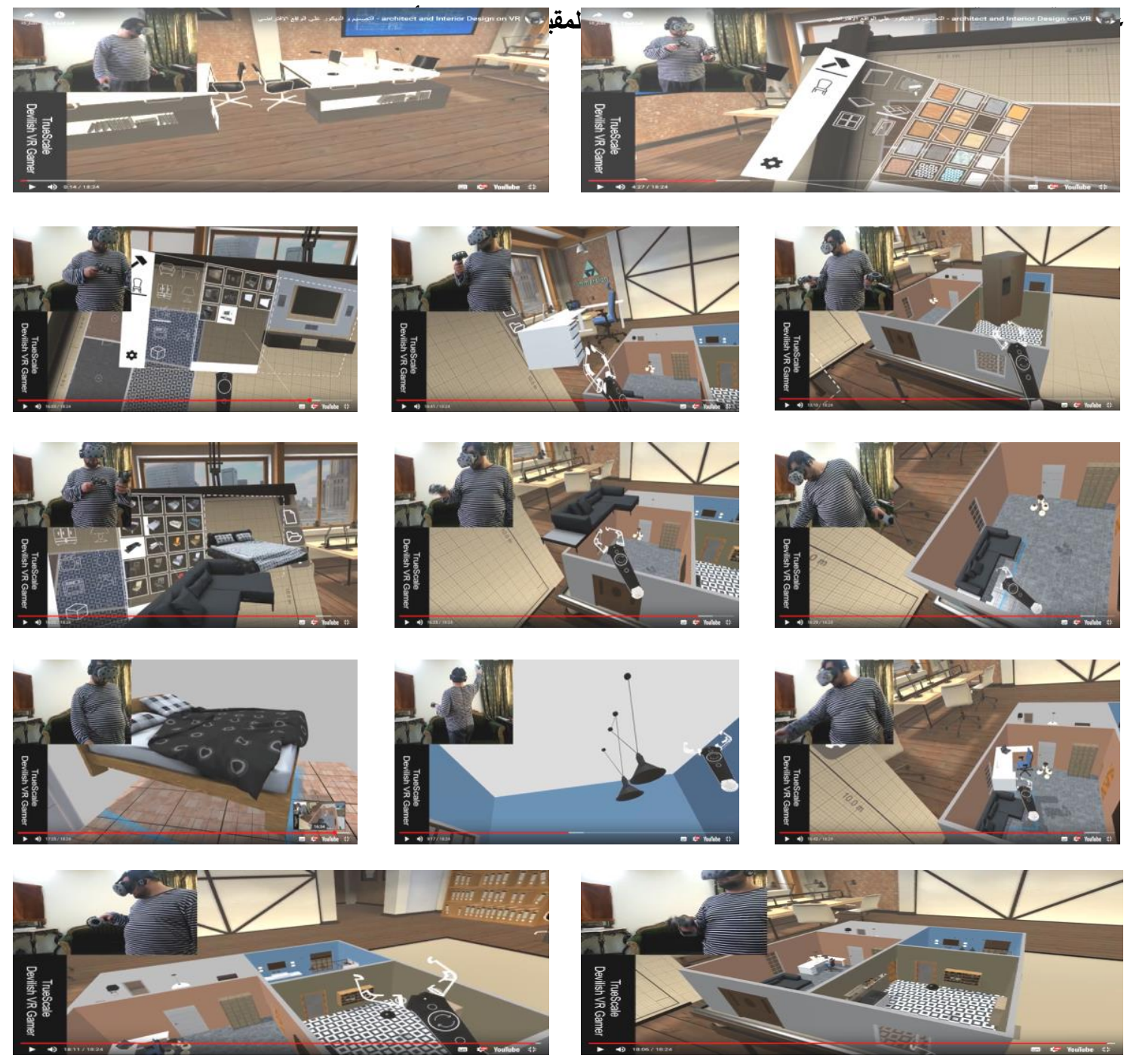
ثَ مستويات أخرى من التفاعل تتثكل بين المستخدم والفراغ، وذلك باعتبار المستخدم في هذه الحالة هو المتلقي والمتفاعل

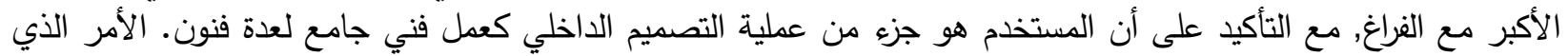

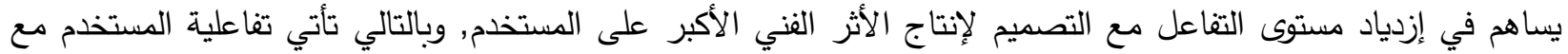

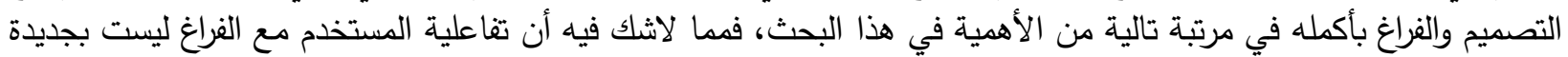
ولكنها تطورت مع ظهور الفراغ الأفتراضي.

ويتأكد هذا المفهوم بالتزامن مع التطور الحالي للفنون الرقمية وتنامى دورها وسيطرة تقنياتها فى تصميم وتنفيذ العمارة الداخلية،

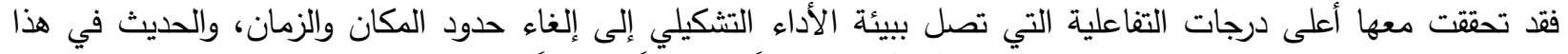

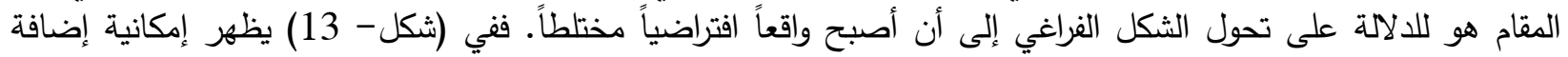

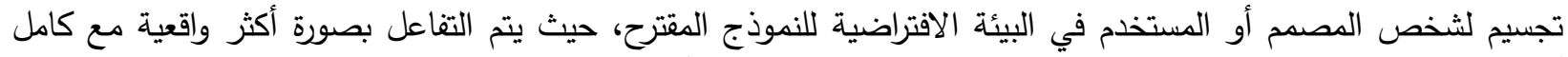

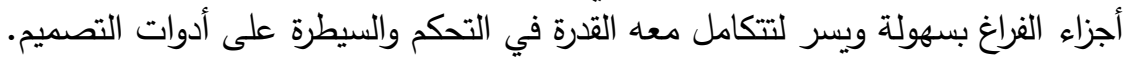

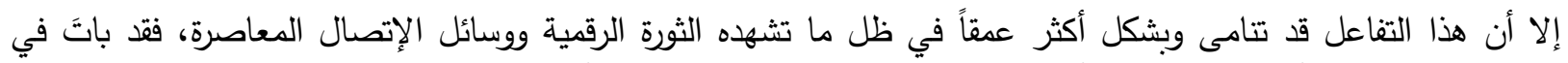

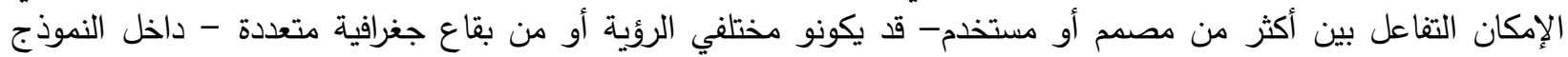

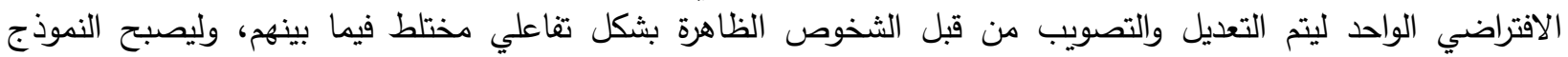
الافتراضي الناتج عبارة عن خليط من الأفكار والرؤى التي باتت تسافر عبر العالم بعيداً عن التصادم المحض لتتفاعل وتتسق فيما بينها في كلٍ فراغي واحد.

( شكل - 13 ) حقق توظيف الواقع الافتراضي المختل لتصور التصميم الداخلي، إمكانيةإضافة تجسيم لشخص المصمم أو
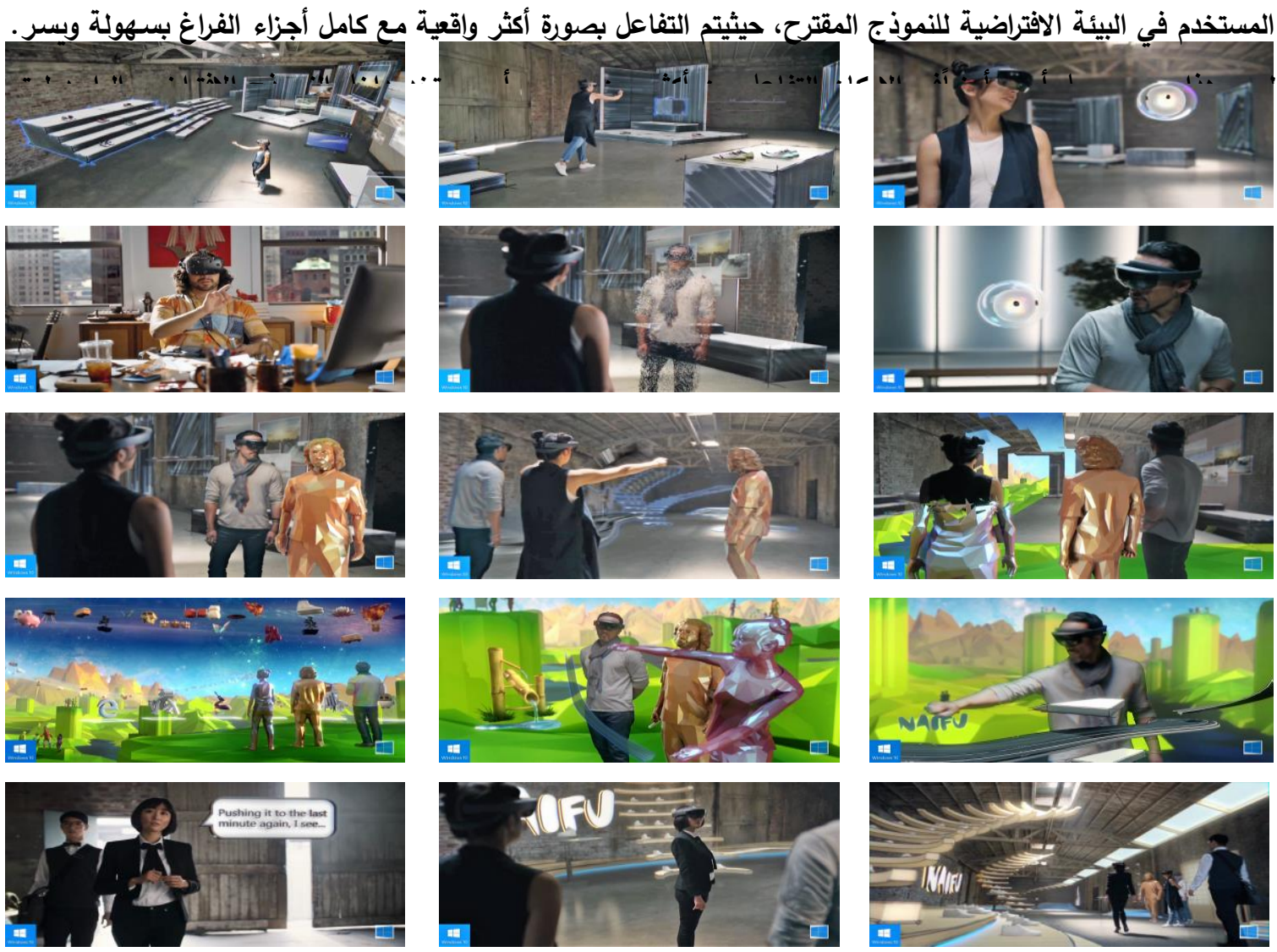


$$
\begin{aligned}
& \text { يسعى الباحث فى الثرح والتحليل لما سبق عرضه من صور ثابتة لنماذج تفاعلية وافتراضية أن ينقل وجهة نظر البحث بالرغم }
\end{aligned}
$$

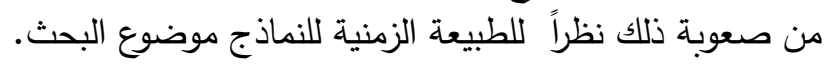

$$
\begin{aligned}
& \text { وقد توصل الباحث إلى مجموعة من النتائج تتركز فيما يلى:- } \\
& \text { 1 - تنامي استخدام التطبيقات الرقمية ساهم في أنتاج علاقات مستحدثة غير محدودة بين مجوعات مختلفة من الفنون } \\
& \text { والفنانين من بينهم فن تصميم العمارة الداخلية. } \\
& \text { 2- أسهمت التقنية الرقمية في الحد من تكلفة التتفيذ وتعاظم قدرات الإبهار في أعمال التصميم الداخلي. } \\
& \text { 3- أن تفاعلية المستخدم مع الفراغ قد تطورت مع تتامي توظيف تطبيقات الواقع الافتراضي في التصميم الداخلي مما زاد } \\
& \text { من تفاعلية البيئة الفراغية. } \\
& \text { 4- أصبح في الإمكان التفاعل بين أكثر من مصمم أو مستخدم داخل النموذج الافتراضي الواحد ليتم التعديل والتصويب } \\
& \text { بشكل تفاعلي مختلط. } \\
& \text { 5- أصبح النموذج الافتراضي التفاعلي عبارة عن خليط من الأفكار والرؤى التي باتت تسافر عبر العالم بعيداً عن } \\
& \text { التصادم المحض لتستق فيما بينها في كلٍ فراغي واحد. } \\
& \text { ولعل في هذه النتائج إجابة على مشكلة البحث والذي افترض أن الفن التفاعلي يمكن أن يصل بالعمارة الداخلية إلى عالم من } \\
& \text { الواقع الافتراضي المختل فذه. } \\
& \text { ويوصى البحث بما يلى:- } \\
& \text { 1- بعد إنفتاح تخصص تصميم العمارة الداخلية على الفنون الرقمية، فإن هناك حاجة ماسة لإعادة النظر في حدود } \\
& \text { التخصص والمتخصصين. } \\
& \text { 2- ضرورة تعديل البرامج الدراسية بما يتلائم مع التطور المبهر والقابل للإستيعاب - إذا توفرت الإمكانات - مع إعادة } \\
& \text { تأهيل وتدريب الكوادر الفنية المصرية. }
\end{aligned}
$$

\section{Abstract}

\section{Interactive art takes interior architecture}

\section{to a world of mixed virtual reality}

The methods of designing interior architecture were not a more continuous and interconnected day, and perhaps even more intertwined than they are now, which created a state of similarity of those methods and the birth of others and may fade some of them, to the extent that confused the user and perhaps the interior designer. It has become necessary for specialists and researchers - both in specialization - to work to resolve this clash and to clarify the reality of this linkage and its limits and the possibility of separation between them, which will allow the designer of internal architecture in the end the opportunity to work and creative conscious productive rather than the prevalence of design work lacks identity, Which will have a positive impact on the user and the entire process of vacuum handling.

In this context, the researcher attempts to focus on the impact of interactive art and its digital applications in the field of virtual reality in general and the specialization of interior architecture 
in particular and the availability of these techniques to reach this specialization to a mixed reality, search. The researcher seeks to prove this hypothesis based on the presentation and analysis of a selected group, the first of the works of interactive art and other of these digital virtual worlds in the internal architecture that contribute to the goal of research.

The problem of research is to answer the following question: Did interactive art in interior architecture transform the spatial form into a virtual reality mixed?

The importance of research is an attempt to add a study in Arabic in the field of interior architecture design, and to shed light on some terms and concepts such as interactive art and concept, digital design or digital presentations, and mixed virtual reality.

The researcher assumes that the application of digital technology in the design and implementation of internal architecture has led to the coloring of this specialization with digital interaction, which led to the emergence of virtual reality in the inner space.

The research aims to prove the hypothesis of research, and answer the question in the problem of research.

Research Methodology-The researcher takes the analytical descriptive approach in conducting this research.

المراجع العربية:

1- أ.د / عصام متولي محمد ) استخدام التقدم التكنولجي في تصميم فراغات داخلية أكثر تفاعلية (كلية العمارة والتصميم - قسم تصميم داخلي، جامعة البطرا، الأردن. المراجع الأجنبية: (T) - (T) - (T)

2- $\quad$ Gates, (The Road Ahead, With Nathan Myhrvold and Peter Rinearson), International Thomson Publishing Inc. New York, 1995.

3- Riewoldt, (Intelligent Spaces, Architecture for the Information Age), International Thomson Publishing Inc. New York, 1997.

مواقع على شبكة الإنترنت:

1- https://www.livescience.com/34843-augmented-reality.html

2- https://www.wired.co.uk/article/ikea-place-augmented-reality-app-space-10

3- https://digiday.com/marketing/ikea-using-augmented-reality/

4- https://gearpatrol.com/2017/07/28/augmented-reality-interior-design/

5- https://www.youtube.com/watch?v=WvBXG1wb36g

6- https://www.youtube.com/watch?v=IP5yeOMlsYQ

7- http://rf102010.blogspot.com/2011/05/virtual-reality.html

8- http://techm3lomat2012.blogspot.com/2012/12/virtual-reality.html

9- https://www.viveport.com/apps/b6a97756-4fce-49aa-a0c5-a111b122ba48 
10- https://www.youtube.com/watch?v=CrBSw7QVAjY

$11-$ https://www.youtube.com/watch?v=1HHhqvFCnAw

12- https://ar.wikipedia.org/wiki/\%D9\%88\%D8\%A7\%D9\%82\%D8\%B9_\%D8\%A7\%D9\%81\%D 8\%AA\%D8\%B1\%D8\%A7\%D8\%B6\%D9\%8A

13- HTC Vive - VR Interior Design Experience - YouTube

14- https://www.youtube.com/watch?v=WMHmkK_PO7I

15- https://www.youtube.com/watch?v=67yLuiSfMWM

16- https://www.youtube.com/watch?v=2MqGrF6JaOM

17- https://www.youtube.com/watch?v=upWRwTbS6bw

18- https://www.youtube.com/watch?v=Oj_x0jvb8w0

19- https://www.youtube.com/watch?v=819qRDMVNRY

20- https://www.youtube.com/watch?v=84QttxTloWI

$21-$ https://www.youtube.com/watch?v=xp1bw7waiFM

22- https://www.youtube.com/watch?v=xTT_3DhTMI8

23- Techm3lomat2012.blogspot.com/2012/12/virtual-reality.html 